\title{
Identification of vessel wall anomalies in thoracic aortic aneurysms through optical coherence tomography and gradient-based strategies
}

\author{
Alma Eguizabal ${ }^{a^{*}}$, Eusebio Real ${ }^{\mathrm{b} * *}$, Alejandro Pontón $^{\mathrm{c}}$, Marta Calvo Diez ${ }^{\mathrm{c}}, \mathrm{J}_{\text {. Fernando Val-Bernal }}$, \\ Marta Mayorga ${ }^{\mathrm{c}}$, José M. Revuelta ${ }^{\mathrm{c}}$, Jose M. Lopez-Higuera ${ }^{\mathrm{b}}$, Olga M. Conde ${ }^{\mathrm{b}}$ \\ ${ }^{a}$ Universität Paderborn (Germany); \\ bUniversidad de Cantabria (Spain); \\ 'Hospital Universitario Marqués de Valdecilla (Spain)
}

\begin{abstract}
Optical Coherence Tomography is a natural candidate for imaging biological structures just under tissue surface. Human thoracic aorta from aneurysms reveal elastin disorders and smooth muscle cell alterations when visualizing the media layer of the aortic wall, which is only some tens of microns in depth from surface. The resulting images require a suitable processing to enhance interesting disorder features and to use them as indicators for wall degradation, converting OCT into a hallmark for diagnosis of risk of aneurysm under intraoperative conditions. This work proposes gradient-based digital image processing approaches to conclude this risk. These techniques are believed to be useful in these applications as aortic wall disorders directly affect the refractive index of the tissue, having an effect on the gradient of the tissue reflectivity that conform the OCT image. Preliminary results show that the direction of the gradient contains information to estimate the tissue abnormality score. The detection of the edges of the OCT image is performed using the Canny algorithm. The edges delineate tissue disorders in the region of interest and isolate the abnormalities. These edges can be quantified to estimate a degradation score. Furthermore, the direction of the gradient seems to be a promising enhancement technique, as it detects areas of homogeneity in the region of interest. Automatic results from gradient-based strategies are finally compared to the histopathological global aortic score, which accounts for each risk factor presence and seriousness.
\end{abstract}

Keywords: Optical Coherence Tomography, aorta aneurysm, wall degradation, gradient-based image processing.

\section{INTRODUCTION}

Cardiovascular diseases related to aortic aneurysms usually require resection of the malformed vessel wall and its replacement by artificial grafts that must be sutured to the remaining patient's aorta [1]. The evolution of the patient after the intervention and the avoidance of post-operative pseudoaneurysm appearance are related to how much vessel tissue is removed and the tissue status on the suture area. The aneurysm risk is conventionally diagnosed through the measurement of the vessel diameter by means non-invasive imaging techniques (ultrasound, computed tomography, etc.), but not always the degradation of a diseased aortic wall is translated into an enlargement of the vessel diameter requiring of an accurate estimation of the current aortic wall degradation.

Under intraoperative conditions, the real-time assessment of the status of the aortic wall would be of great help for cardiovascular surgeons. Conventional histopathological techniques for wall assessment (Hematoxylin and Eosin, Verhoeff's Van Gieson, Alcian Blue and Alpha-smooth muscle actin stains) become not suitable for real-time diagnosis as they require of tissue extraction and batch processing. Human thoracic aorta from aneurysms reveal morphological changes (elastin disorders and smooth muscle cell alterations) when visualizing the media layer of the aortic wall [2] which is only some tens of microns in depth from surface. In this scenario, OCT (Optical Coherence Tomography) becomes a natural candidate for imaging the inner structure of the aortic wall under real-time conditions where no stains of cuts are needed.

\footnotetext{
*alma.eguizabal@sst.upb.de; phone +49 (0)5251 60-5381; fax +49 5251 60-2989; sst.upb.de

**eusebio.real@unican.es; phone +34 942 200877+12; gif.teisa.unican.es
} 
Tissue changes in the aortic wall structure appear as changes in the reflectivity tissue profile imaged by the OCT technique. Under this circumstances, gradient-based techniques [3][4] are useful to determine the edges of the areas where tissue presents anomalies or changes on its morphological organization. An automatic estimation of degradation can be assessed studying the evolution of the gradient (magnitude and direction) of the reflectivity profile. The gradient magnitude seems to be related to how different is the tissue in each layer of the aortic wall, whereas the magnitude direction comes to reveal tissue areas with uniform behavior. A combined analysis of both approaches will provide an estimation of the degradation of the aortic wall in the real-time conditions needed for intraoperative assessment converting OCT in a viable technique for optical biopsy in the cardiovascular context.

\section{MATERIALS AND METHODS}

\subsection{Aortic samples and Optical Coherence Tomography set-up}

Human ex-vivo aorta specimens come from two sets of aortas. The first set consists of 3 healthy aorta specimens, corresponding to donors for heart transplantations. The second set consists of 12 degraded specimens coming from Ascending Thoracic Aorta Aneurysm interventions. Samples were collected during surgery intervention and conserved refrigerated in phosphate buffered saline before being measured with the OCT system. Each aortic sample was divided into different regions of interest along the vessel circumference, intended to provide various points for contrasted characterization within the Ascending Thoracic Aorta region.

OCT measurements were performed with Thorlabs OCS1300SS system. This system is based on a $1325 \mathrm{~nm}$ swept source and has a resolution in air of $12 \mu \mathrm{m}$ axial and $25 \mu \mathrm{m}$ transverse. Measurements were taken focusing the probe on the surface of the sample, corresponding to the intima layer of the aorta artery [5]. Several cross-sectional images (Bscans) are taken on every region.

After OCT measurements, examined tissue was conserved in $10 \%$ formalin solution for a further histopathological analysis performed by expert pathologists. Different staining techniques were applied to assess the condition of the vessel wall and its constituents: Hematoxylin and Eosin and Verhoeff's Van Gieson for elastic fibers evaluation, Alcian Blue for acidic polysaccharides and Alpha-Smooth Muscle Actin for smooth muscle cells.

Histological information is considered the reference of the specimen true condition. After this characterization, a semiquantitative grading scheme is used to precisely characterize degradation of the analyzed samples [2]. This grading scheme categorizes 5 different structural deformation scores from 0 to 3 according to the physical extension of abnormalities in the media layer of the artery. Summation of the previous scores gives a total quantification of deformation from 0 (completely healthy artery) to 15 (severely degraded artery).

According to the histopathological information, artery wall degradation is evidenced in the media layer. Thus, the analysis of obtained data is centered on that region of interest. In order to analyze OCT images properly, it is necessary a pre-processing to segment this region of interest in every B-scan. First of all, Otsu's segmentation algorithm [6] is used to automatically differentiate the air above the surface from tissue. The second step is to limit the inferior part of the Bscan, corresponding with low reflected intensity due to tissue absorption. This region is limited to 150 pixels below the surface, according to statistics from the measured samples [5].

\subsection{Gradient-based image processing strategies}

In this section we describe some well-known image processing strategies designed to perform image enhancement in the spatial domain. Here, the spatial domain term refers to the image plane. Therefore, these approaches directly manipulate the pixels of the image on their spatial location to find more meaningful information for the current application [7].

Firstly, to compute spatial domain enhancement, a mask is designed (also referred as template, window or kernel) which is generally small (i.e. $3 \times 3$ pixels) and squared. This mask behaves as a sub image whose size defines a neighborhood of operation. Its center is moved pixel by pixel, sliding through the whole image, to determine a new value for each one of the pixels, using only the values inside the neighborhood to compute this new value. This process is known as convolution between the mask and the image.

Giving the nature of OCT images from aorta tissue, calculating relative changes between pixels becomes an issue. An estimation of these changes can be easily computed using linear operators in the spatial domain. These are the so-called Gradient Operators, which estimate the first derivative of an image and can be easily computed using some specific 
masks. A gradient operator will permit to highlight fine details, as the strength of the response of a derivative is proportional to the degree of discontinuity at the point at which the operator is applied. Thus, a computation of differences, i.e. an estimation of the derivative of the image, will enhance edges and other discontinuities within the layers that might be associated with the pathology important to the application.

\subsubsection{Derivative estimation by gradient operators}

An image can be considered a function $f(x, y)$, whose gradient is defined as the following two-dimensional column vector:

$$
\nabla f=\frac{\partial f}{\partial x} \hat{x}+\frac{\partial f}{\partial y} \hat{y}
$$

This can be computed using the first derivative in the horizontal direction $G_{x}$ and the vertical direction $G_{y}$ are also defined as:

$$
\mathrm{G}_{\mathrm{x}}=\frac{\partial \mathrm{f}}{\partial \mathrm{x}} \text { and } \mathrm{G}_{\mathrm{y}}=\frac{\partial \mathrm{f}}{\partial \mathrm{y}}
$$

Considering the image is a matrix containing gray-level values (reflectivity levels in OCT images), the x-axis is defined along the columns of the images going right (along the aortic surface of the intima layer), and the y-axis is defined along the rows going down (the thickness of the aortic wall from the intima to the media layer). Therefore, two different kernels must be defined to obtain the orthogonal components of derivative, $G_{x}$ and $G_{y}$, and perform a digital approximation to the preceding equations. In order to a better understanding of these gradient templates, Figure 1 presents an an example of a filter mask.

\begin{tabular}{|l|l|l|}
\hline$z 1$ & $z 2$ & $z 3$ \\
\hline$z 4$ & $z 5$ & $z 6$ \\
\hline$z 7$ & $z 8$ & $z 9$ \\
\hline
\end{tabular}

Figure 1. Example of a $3 \times 3$ generic mask, where the pixel in the center, $\mathrm{z} 5$, is the one whose gradient is being calculated using the values of the rest of the pixels in the mask.

Assuming the operation is over the center pixel, $z 5$, the simplest approximation to a first-derivative would be to compute an intermediate difference, i.e. $\mathrm{G}_{\mathrm{x}}=(z 8-z 5)$ and $\mathrm{G}_{\mathrm{y}}=(z 6-z 5)$. Also, a different definition was proposed by Roberts using cross differences, $\mathrm{G}_{\mathrm{x}}=(z 9-z 5)$ and $\mathrm{G}_{\mathrm{y}}=(z 8-z 6)$, which highlight the edges.

Nevertheless, all these approaches would need a mask of even size to be performed, and the indetermination of the center point of analysis can be a drawback, as it shifts the result by half a pixel. Thus, templates using a $3 \times 3$ size have also been defined computing $\mathrm{G}_{\mathrm{x}}=(z 7+\mathrm{c} * z 8+z 9)-\left(z 1+c^{*} z 2+z 3\right)$ and $\mathrm{G}_{\mathrm{y}}=(z 3+c * z 6+z 9)-\left(z 1+\mathrm{c} *^{*} 4+z 7\right)$. This is the case in Prewitt and Sobel operators, where Prewitt has a constant value $c=1$ while in Sobel $c=2$ to achieve smoothing by giving more importance to the center point. Examples of these templates are shown in Figure 2. 
(a)

\begin{tabular}{|l|l|}
\hline+1 & 0 \\
\hline 0 & -1 \\
\hline
\end{tabular}

\begin{tabular}{|l|l|}
\hline 0 & +1 \\
\hline-1 & 0 \\
\hline
\end{tabular}

(b)

\begin{tabular}{|c|c|c|}
\hline-1 & -1 & -1 \\
\hline 0 & 0 & 0 \\
\hline+1 & +1 & +1 \\
\hline
\end{tabular}

\begin{tabular}{|l|l|l|}
\hline-1 & 0 & +1 \\
\hline-1 & 0 & +1 \\
\hline-1 & 0 & +1 \\
\hline
\end{tabular}

Figure 2. Gradient templates: (a) Roberts templates, $G_{x}$ (left) and $G_{y}$ (right), consider the cross-difference but are $2 x 2$ and shift the result half a pixel; (b) Prewitt operators, $\mathrm{G}_{\mathrm{x}}$ (left) and $\mathrm{G}_{\mathrm{y}}$ (right) do not present this drawback.

Once the orthogonal components of the gradient are obtained, $G_{x}$ and $G_{y}$, the magnitude and the direction of the gradient is calculated straightaway following (3):

$$
\text { magnitude }=\left|\mathrm{G}_{\mathrm{x}}\right|+\left|\mathrm{G}_{\mathrm{y}}\right| \quad \text { direction }=\tan ^{-1}\left[\frac{\mathrm{G}_{\mathrm{y}}}{\mathrm{G}_{\mathrm{x}}}\right]
$$

Furthermore, before performing the gradient operators, a smooth of the image is usually performed, i.e. a Gaussian filter convolution or a median filter strategy. This may eliminate noise and give more uniformity to the image preserving the structure of the edges.

\subsubsection{Edge detection using Canny algorithm}

A simple way of extracting edges from an image after estimating the gradient would be just to threshold the magnitude of the gradient, in order to obtain its maximum response, which would correspond to the highest gray-level changes, i.e. the edges of the image. However, the resulting image might be too crowded of edges and it would also be difficult to determine a correct threshold to preserve the structural interesting properties. Thus, Canny [8] developed an algorithm that is optimal regarding the detection of the edges and its localization, making used of both the magnitude and the direction of the gradient. Most important steps of the algorithm are the following:

- Non-maximum suppression: only those pixels with highest gradient magnitude in the direction of the gradient are kept, as highest magnitudes should occur right at the edge boundary direction. The pixel is only preserved as edge if it has the highest magnitude within the neighborhood defined by gradient direction.

- Hysteresis thresholding: a simple threshold may remove valid parts of a connected edge if it is too high and also may consider noise pixels as valid edges if it is too low. Thus, two thresholds (one high and one low) are used. Pixels with weaker gradient magnitude than the low threshold will be directly discarded. However, pixels between high and low thresholds are only kept if they form a continuous edge line with pixels with strong amplitude (i.e. the ones with stronger amplitude than the high threshold). These thresholds may depend on the statistical moments of the image in analysis, i.e. the mean gray-value, the maximum gray-value or the interquartile ratio.

\section{RESULTS AND DISCUSSION}

The goal of this study is to verify that an enhancement processing based on gradient operators over the OCT images of aorta samples is suitable to determine the degradation state of the aorta wall. The pre-processing stage [5] permits to obtain a region of interest, isolating the intima and media layer, as well as eliminating the ribbon-of-air above the aortic surface. Also, the ribbon-of-air pre-detection permits a rotation of the image when this is not aligned, using the horizontal limits of the ribbon and a trigonometric rule to define an angle of rotation; finally a nearest neighbor interpolation is used after the affine transformation. This way, the gradient operators are computed always over images in the same position. 
The first decision is to determine which gradient operator (Roberts, Prewitt or Sobel) would be more suitable for the application. We verified that $3 \times 3$ templates give more contrast to the potential edges than $2 \times 2$ approaches. Figure 3 shows a B-scan of an aneurysmatic aortic wall where Prewitt (Fig.3.d) provides an enhanced image of the tissue anomalies when compared with a Roberts strategy (Fig.3.c) to estimate $\mathrm{G}_{\mathrm{x}}$ and $\mathrm{G}_{\mathrm{y}}$. The abnormalities of this particular sample correspond to elastic fragmentation, which is one of the features that the gradient-based analysis will enhance.

(a)

(c)

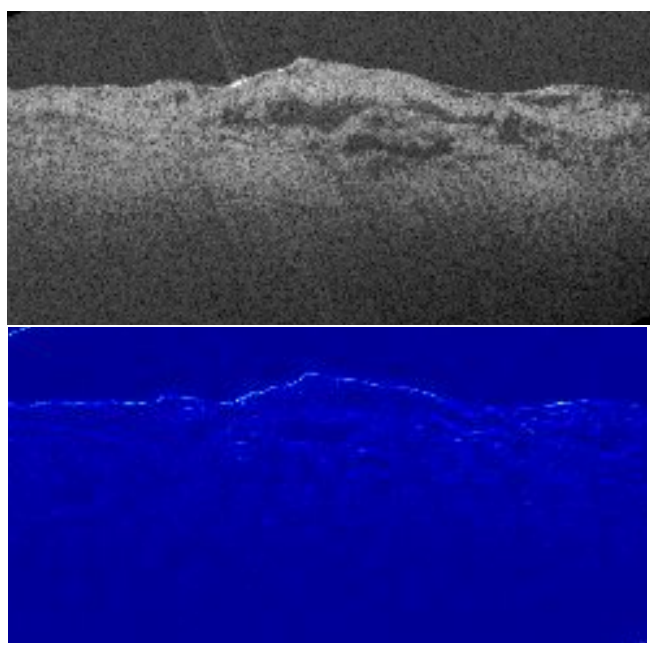

(b)

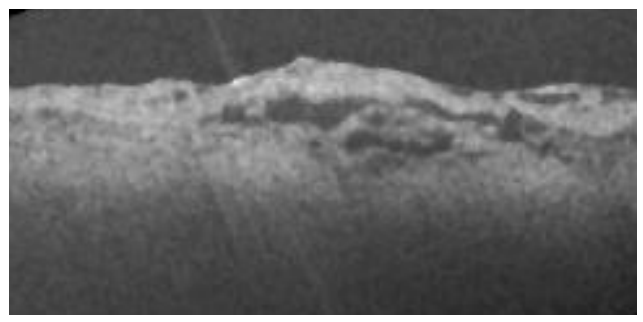

(d)

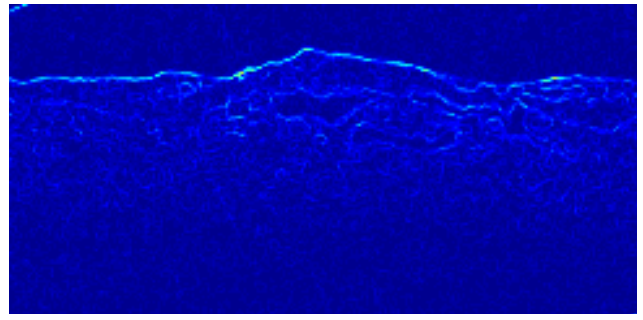

Figure 3. OCT image of an aneurysmatic aortic wall (pathological score=10): (a) original B-scan image; (b) B-scan image after a median filter to delete measurement noise preserving edge structure; (c) gradient magnitude using Roberts operator; (d) gradient magnitude using Prewitt operator. In both (c) and (d) lighter colors correspond to higher values of the magnitude

From the intermediate gradient results, $\mathrm{G}_{\mathrm{x}}$ and $\mathrm{G}_{\mathrm{y}}$, interesting features for the analysis have been preliminarily observed in the direction of the gradient. Figure 4.c shows the gradient orientation image, where the noisy areas correspond to those uniform regions with low SNR (air, deep tissue...); in these regions the magnitude of the gradient is also low so the direction actually is not well defined from a numerical point of view and therefore for its interpretation. Then, a low pass filtering based on a Gaussian convolution with a $25 \times 25$ kernel size in the space domain is used, to result on the smoothed version of the gradient orientation showed on Fig.4.d. Here some features that correlate with the presence of homogeneous areas in tissue are observed.

(a)

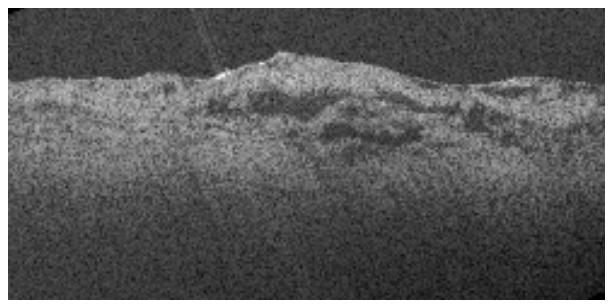

(c)

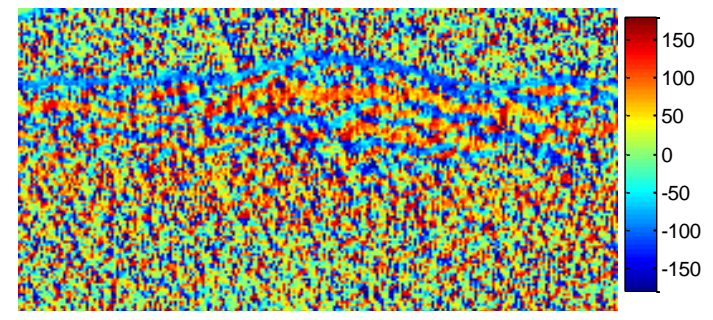

(b)

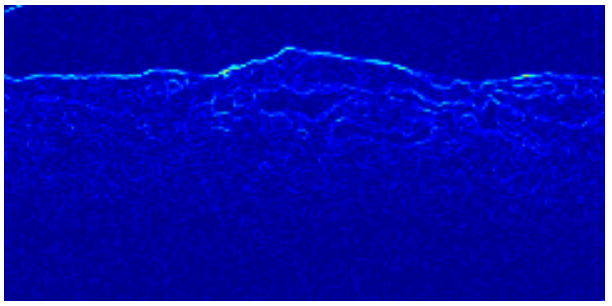

(d)

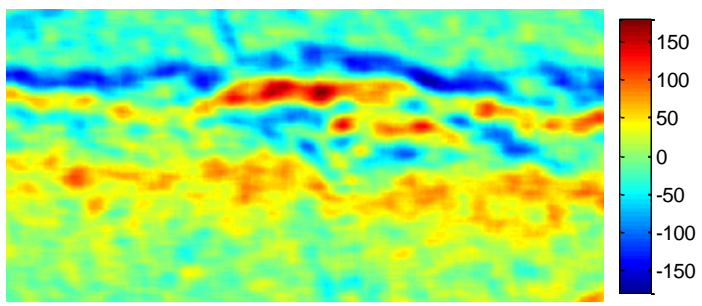

Figure 4. An example of (a) original image, (b) gradient magnitude (bright colors are the highest), (c) gradient direction and (d) low-pass filtered gradient orientation. It is observed that (d) presents interesting features for the pathological analysis as it highlights areas of tissue homogeneity. 
For that reason, two paths of analysis have been developed from this point:

(a) The use of gradient magnitude and direction using Prewitt operator to perform a Canny edge detector and obtain the edges in the region of interest. Aorta tissue with abnormalities associated with aneurysm will have more edges, as the edge detector will work to isolate these irregularities

(b) The use of a smoothed version of the direction of gradient obtained with Prewitt operator, as it seems to be a hallmark of the tissue uniformity itself. Healthy-sample images present more homogenous tissue, so the direction of the gradient will be uniform in the region of interest.

\subsection{Results based of Canny edge detector}

It is been observed that, as healthy samples present fewer anomaly structures in their media layer, more homogenous OCT images are generated. Then, the edge detector hardly detects borders within the region of interest. The quantification of the number of edges permits an alternative estimation of the histopathological score by means OCT. Figure 5(a)(b)(c) shows OCT images measured from tissue coming from a healthy aorta with pathological degradation score 1, and Figure 5(d)(e)(f) from an aneurysmatic aorta whose pathological degradation has been quantified as 12 . As observed in Fig.5.b (healthy sample) and Fig.6.e (aneurysmatic sample), edges (in red) tend to appear around the abnormalities typical from diseased samples, corresponding to elastin disorders and muscle cell alteration. This fact allows using the quantification of edges as an estimate of the pathological score. Results of the relation between this score and the edge-quantification parameters are shown in Fig.6.a.

\subsection{Results based on the direction of the gradient itself}

As discussed above, it is been detected that the direction of the gradient contains valuable information to enhance the uniformity or irregularity of an OCT image. After a low-pass filtering of the gradient direction, areas of homogeneity in the media layer tend to keep a direction close to $180^{\circ}$. A quantification of these areas reveals the histological state of the sample, this time without using any edge detector afterwards. Results on a healthy and aneurysm sample are shown in cyan in Fig.5.c and Fig.5.f respectively, where bigger areas of homogeneity are found in control samples. Also the correlation of this feature with the histopathological score is shown in Fig.6.b.
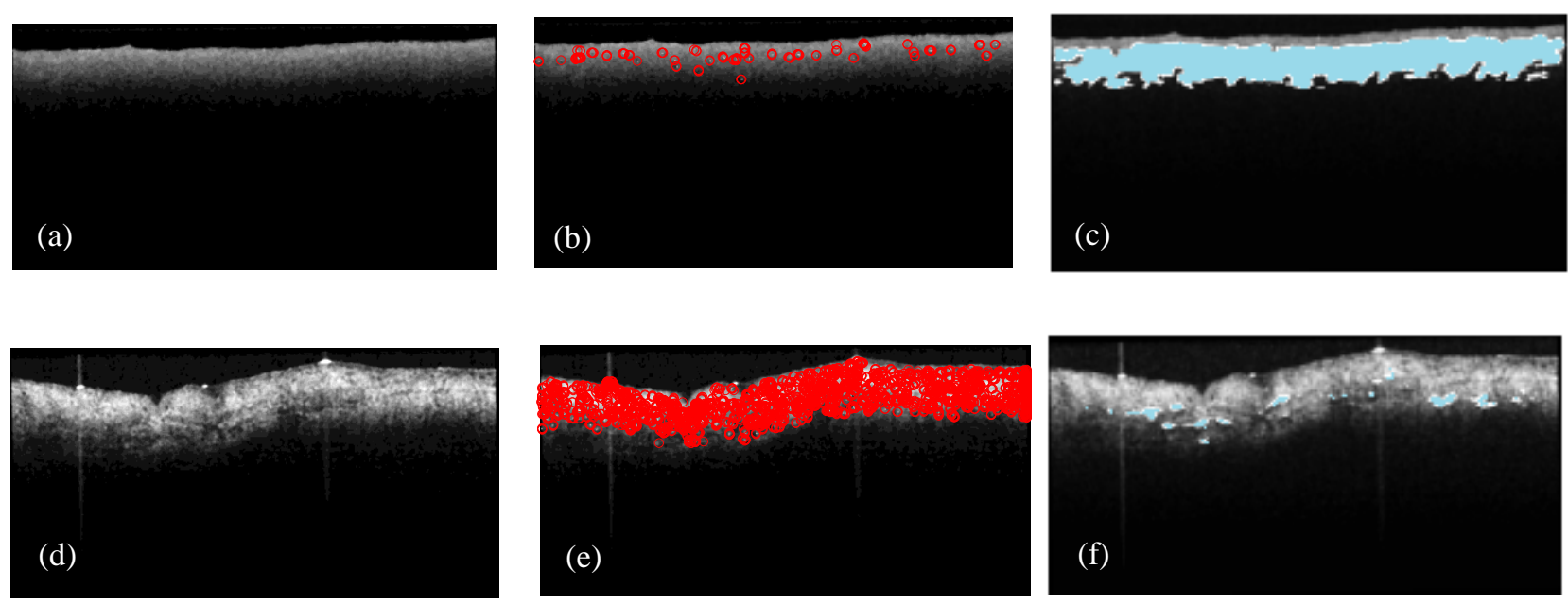

Figure 5. OCT images from healthy aorta (pathological score 1): (a) original OCT image; (b) edges quantification in red and (c) homogeneity areas (in cyan) after a threshold of the gradient orientation. Then, OCT images of an aneurysmatic aorta (pathological score 12): (d) original OCT image; (e) edges quantification in red and (f) homogeneity areas (in cyan) after a threshold of the gradient orientation. 


\subsection{Comparison with the pathological score}

As discussed above, a set of samples has been evaluated to study the relation with the gradient features and the pathological score. A compacted view of the results is shown in Fig.6. As expected, the pathological score of degradation is directly correlated with the number of edges found after the Canny processing of the gradient (Fig.6.a). On the other hand, the size of the areas of uniformity in tissue decays proportionally to the degradation of the aortic wall (Fig.6.b).

\section{Canny edges}

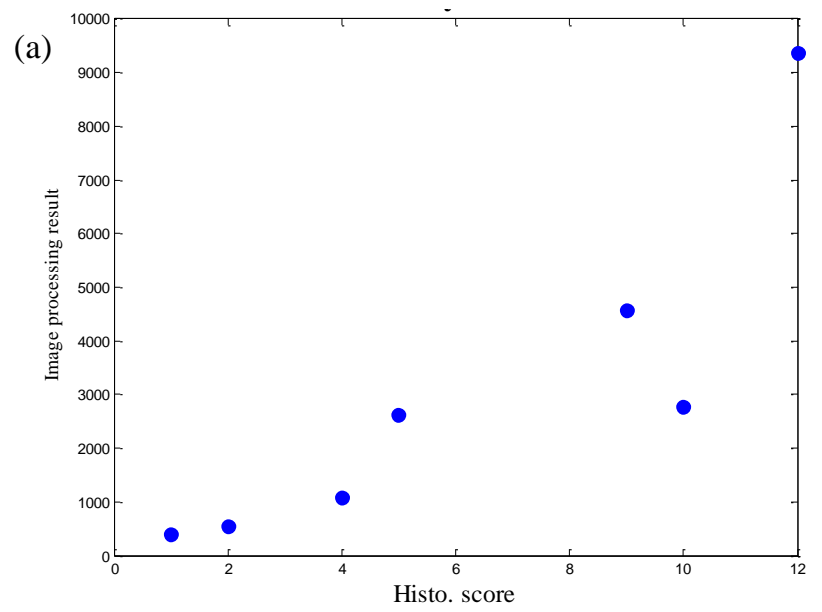

Direction homogeneity

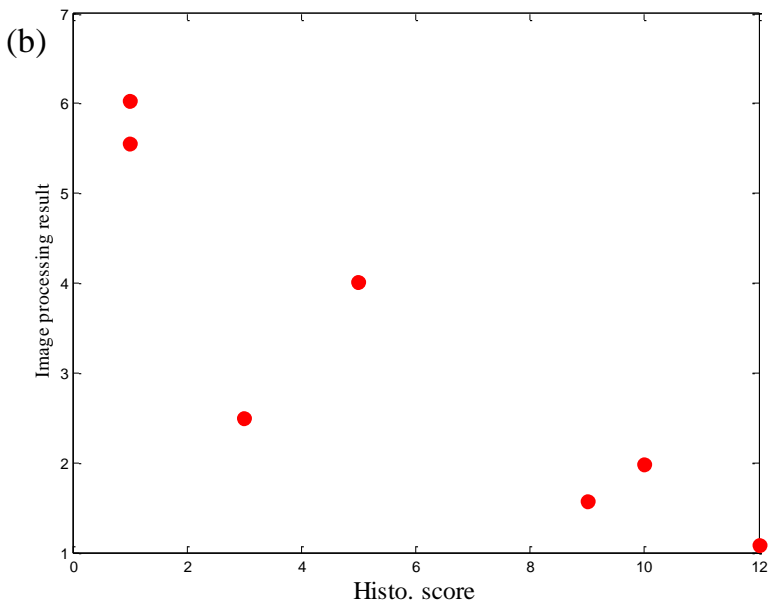

Figure 6. Validation of the OCT-based degradation score when compared with the histopathological score: (a) OCT-metric based on the quantification of edges; (b) OCT-metric based on the quantification of homogeneous areas from gradient direction.

\section{CONCLUSIONS}

Optical Coherence Tomography is presented as a real-time alternative to traditional histology when open surgery of cardiovascular aortic repair is performed. Sometimes, OCT technology does not produce images with relevant diagnostic contrast and an enhancement image processing is required to improve the usability of the OCT measurements.

Human ascending thoracic aorta from aneurysms reveals elastin disorders and smooth muscle cell alterations when visualizing the media layer of the aortic wall. Isolating this region of interest is the first step. Then, gradient operators have been proposed to highlight the pathological information. Two different and complementary interpretations of the gradient have been considered. Firstly, the gradient magnitude and direction are employed to perform a Canny edge detection assuming that aneurysmatic samples will show more edges surrounding the abnormalities of the tissue. On the other hand, a post-processed version of the gradient direction reveals areas of homogeneity associated to the presence of uniform tissue that becomes larger in case of healthy aortae.

Results are promising so far as both proposed interpretations correlate quite well with the semi-quantitative histopathological indicator [2]. There are evidences of a relation between the histopathological score of the sample and the image processing results obtained from the gradient of the image. Nevertheless, this process is subject to be optimized. A design of an automatic detection will be performed in order to estimate the detection rate of a classifier based on the gradient features obtained. In addition, many parameters of the approach can still be optimized, i.e. size of preprocessing filters, thresholds to define areas of homogeneity etc. This stage will be performed with an exhaustive search. 


\section{ACKNOWLEDGMENTS}

This work has been supported by the project DA2TOI (FIS2010-19860) and Alma Eguizabal's SPIE Optics and Photonics Education Scholarship.

\section{REFERENCES}

[1] Bentall, H., De Bono, A., "A technique for complete replacement of the ascencing aorta," Thorax, 23(4), 338-339 (1968).

[2] Bechtel, J. F. M et al., "Histopathological grading of ascending aortic aneurysm: comparison of patients with bicuspid versus tricuspid aortic valve," J. Heart Valve Dis. 12(1), 54-61 (2003).

[3] Q. Yang, C. Reisman, Z. Wang, Y. Fukuma, M. Hangai, N. Yoshimura, A. Tomidokoro, M. Araie, A. Raza, D. Hood, and K. Chan, "Automated layer segmentation of macular OCT images using dual-scale gradient information," Opt. Express 18(20), 21293-21307 (2010).

[4] Alonso-Caneiro, D., Read, S.A., Collins, M.J., "Automatic segmentation of choroidal thickness in optical coherence tomography", Biomed Opt Express 4(12), 2795-2812 (2013).

[5] Real, E., Eguizábal, A., Pontón, A., Calvo-Díez,M., Val-Bernal, J. F., Mayorga, M., Revuelta, J.M., López-Higuera, J.M. and Conde, O.M., "Optical coherence tomography assessment of vessel wall degradation in thoracic aortic aneurysms," J Biomed Opt 18(12), 126003-126003 (2013).

[6] Otsu, N.,"A Threshold Selection Method from Gray-Level Histograms," IEEE Sys Man Cyb 9(1), 62-66 (1979).

[7] Gonzalez, R. C. and Woods, R. E., [Digital Image Processing], Prentice Hall, New Jersey, 134-137 (2008).

[8] Canny, J., "A computational approach to edge detection," IEEE Transactions on Pattern Analysis and Machine Intelligence 8(6), 679-698 (1986). 